ESAIM: PROCEEDINGS AND SURVEYS, December 2014, Vol. 47, p. 36-54

F. Abergel, M. Aiguier, D. Challet, P.-H. Cournède, G. Fä̈, P. Lafitte, Editors

\title{
MULTISCALE MODELING, STOCHASTIC AND ASYMPTOTIC APPROACHES FOR ANALYZING NEURAL NETWORKS BASED ON SYNAPTIC DYNAMICS
}

\author{
D. HOLCMAN ${ }^{1}$
}

For encouraging adventurous mathematicians to work on novel theoretical challenges arising from the biological sciences.

\begin{abstract}
How do neurons coordinate in complex networks to achieve higher brain functions? Answering this question has relied on experimental approaches based on functional imaging, electrophysiology and microscopy imaging, but surprisingly, what is now really missing in order to make sense of large data are analytical methods, multiscale modeling, simulations and mathematical analysis. Studying neuronal responses while accounting for the underlying geometrical organization, the details of synaptic connections and their specificity remains great challenges. With more than $10^{11}$ neurons, connected by thousands of synapses per neuron, it is not clear what is the right modeling, for bridging the multiple scales starting at nanometer with the release of thousands of diffusing neurotransmitters molecules around synapses, leading to a signal that ultimately integrates into a neuronal network response (few millimeters) resulting in computations that underlie higher brain functions.

We discuss here recent progress about modeling and analysis of small and large neuronal networks. We present neural network modeling based on synaptic dynamics. The models are formulated as stochastic differential equations. We focus on the time-response of the network to stimulations. These time responses can be analyzed as an exit problem of a stochastic trajectory from the basin of attraction, the dynamics of which presents novel characteristics: the attractor is located very close to the separatrices. This property leads to novel phenomena, manifested by oscillatory peaks of the survival density probability. Finally, this report illustrates how mathematical methods in neuroscience allows a better understanding of neural network dynamics.
\end{abstract}

\section{INTRODUCTION}

We still do not understand how local neural networks process information underlying higher brain functions, such as language, cognition or memory. There is still a huge gap between the dynamics of a single neuron and the colorful picture of fMRI images representing the brain in action, obtained by monitoring tens of millions of neurons. Understanding how computations are integrated from a single neuron to millions is a neuroscience hurdle and an adventurous mathematical challenge, which may become in thirty years part of pure mathematics. But we are still far from that point and from bridging the gaps between the multiple scales involved in this process. Starting with the phenomenological description of neuronal networks, we will continue with some mathematical analysis of mean-field models and methods to compute responses generated by many connected

1 Ecole Normale Supérieure, Applied Mathematics and Computational Biology, IBENS/CNRS/INSERM, 75005 Paris, France.

This research reflects BioNewmetrics.com effort.

(c) EDP Sciences, SMAI 2014 
neurons. We will discuss mathematical challenges that we recently found in analyzing these models. Elucidating the phase space of a model for example is usually a challenge that allows exploring the dependency of many parameters and may result in new understanding and predictions. We will adopt this strategy to study neuronal networks and to examine their refined properties. In the following paragraphs, we recall some classical background of neurobiology.

During development, neurons connect and organize in the mammalian brain in a very reproducible and programmed manner, the rules of which are still unknown [27]. Although changes occur during the entire life, once neuronal connections are formed, evolved brain functions are generated, such as long and short term memory, processing of complex sensory stimulations and many others [27]. So what is the basis of such connections, what is specific to neurons that allows information processing?

Although today, there are no specific answers, we shall say that certainly the key of decoding the neuronal code is imbedded in neuronal organization, ordered at different scales in sub- and specialized regions such as the hippocampus, cortical areas, visual cortex and the auditory cortex. Hubel and Wiesel Nobel prices in 1965 rewarded methods and findings demonstrating that single neurons could fire Action Potential (AP) in response to complex stimulus, such as moving bar in a given direction but not the opposite one. Another example is that a rotating bar generates in the visual cortex a rotating activity around singular points (in the topological sense) defined as pinwheels $[18,36]$. The neuronal organization of the visual cortex has been well modeled using interactions between inhibitory and excitatory neurons with synaptic strength connections, modeled by a Mexican hat function (derivative of a Gaussian) [36,38]. These examples show that a single neuron connected to its network can respond to an identified stimuli and this property is a consequence of the network behavior. Let us summarize the phenomenology underlying neuronal communication: neuron can generate APs, which are large amplitude and robust and local perturbation (depolarization) of the membrane potential (of the order of milliseconds in time). Such an AP or a spike can activate synapses. Indeed at chemical synapses, which are connections between neurons, the AP can generate in connected neurons, a local change of the membrane potential (a depolarization). A summation of many depolarization can then generated an AP that will propagate in the connected neurons, leading to the propagation or processing of information in the extended neuronal network. This generation of APs can be spontaneous or generated by external stimulations. The amplitude of the depolarization following a synaptic activity defines the synaptic weight. The modulation of such weight is understood as synaptic plasticity, the basis of learning and memory. In that context, what are the possible signals that can be generated in neuronal ensembles? Fast oscillations, periodic signal, wave propagating in the retina, bursting like seizure or epilepsy have been reported, see also [27] and the classification is certainly not terminated today.

We shall focus our subject on the activity generated in small ensembles containing 5 to 50 neurons, forming islands with many synaptic recurrent connections [29,51]. These small neuronal networks can generate complex patterns of activity, mostly driven by the dynamics of functional synapses. We know how to derive dynamical systems that can reproduce a single stimulus in these neuronal ensembles, which generates a synchronous longlasting bursting activity $[9,10]$. Interestingly, in vitro, a single spike triggered a reverberating network activity that lasted 2-5 seconds. A second interesting phenomenon that we shall discuss here is the spontaneous activity in neuronal networks connected by synaptic dynamics. They can sustain long periods of membrane potential depolarization, lasting for hundreds of milliseconds. In addition, due to the noise, the neuronal membrane potential can switch back and forth between an Up- to a Down- state (depolarized state). This phenomena was modeled using synaptic-depression model [14,23] and we propose to focus here on the distribution of times in the Up-state. Analyzing the model equation reveals that this distribution has some surprisingly oscillatory peaks. These peaks are associated to the second eigenvalue of the Fokker-Planck operator of the stochastic equations. The spectrum of this nonself-adjoint operator can be explicitly computed and we will show that the distribution of times in the Up-state does not follow a Poissonian law. By exploring the phase space, similar approaches have been used to study the interspike distribution driven by noise [48]. In summary, recent models of neuronal dynamics revealed a novel phenomenology, that extends the traditional escape problem [19,33] and requires novel analytical tools. 
This progress report is organized as follows: in the first section, we recall some elementary concepts about neural network physiology and synaptic modeling. In section 2 and 3, we present bursting reverberation and the spontaneous activity in neuronal networks. In section 4, we present a novel analysis of the Fokker-Planck equation used to study the genesis and the consequences of spontaneous activity in neuronal networks. The mathematical model is based on peculiar stochastic dynamical systems. The analysis involves computing the spectrum of non-self-adjoint operators and estimating the solutions requires a refined WKB expansion.
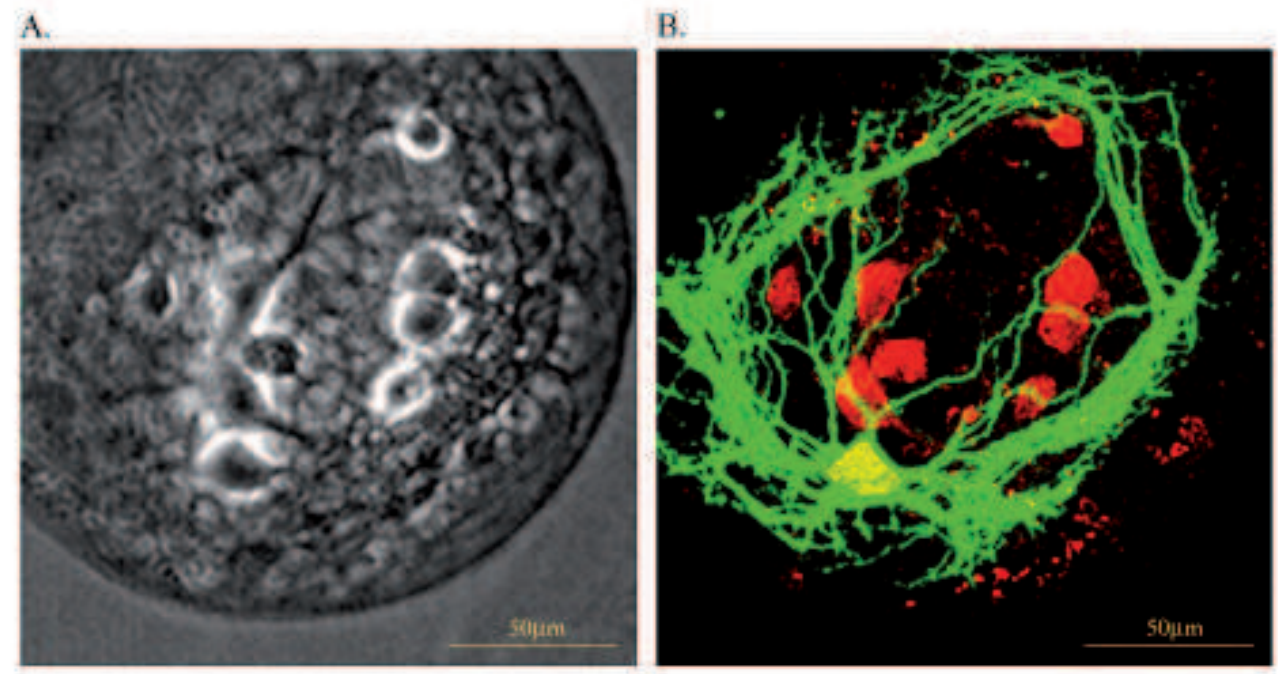

Figure 1. Small neuronal islands. In A. and B. islands are made of few neurons (5 to 30). The cell are filled with biocytin for immunohistochemical identification (red). The island was co-stained with synaptophysin (green dots) showing synapses in the network.

\section{NEURAL NETWORK PHYSIOLOGY: DESCRIPTION AND PHENOMENOLOGY}

Organized and synchronized intrinsic neural activity in neuronal network or cerebral cortex exists even in the absence of any external stimuli $[12,28,47]$. In the auditory cortex for example, intrinsic neural activity is monitored by electro-encephalogram (EEG) recordings. But, identifying the neural organization responsible for patterns of activity remains a daunting hurdle. This activity depends both on intrinsic biophysical and synaptic properties of neurons in the network. The biophysical properties of neurons originates from channel dynamics (see Hille's book [22]).

Recently, it was found that stimulating a single neuron from a connected ensemble can generate a burst activity, called bursting reverberation $[20,42]$ characterized by a long (few seconds) electrical discharge. However, the properties of synchronized neuronal network underlying these bursts are still not fully understood [29,51]. Earlier studies proposed that membrane currents generated by calcium and calcium-gated potassium channels create plateau potentials, which can depolarize neurons for seconds $[37,40]$. On the other hand, synaptic properties are essential for the generation and maintenance of the bursts [3,9,41]. Recent studies proposed that specific 'hub' long range inhibitory neurons in the hippocampus are the ones to trigger and synchronize network bursts [39]. In hippocampal neurons grown in microcultures [9], network bursts induced by a single action potential triggered in one neuron of the network, reverberate for several seconds. This reverberating burst is followed by a long refractory period, assumed to be caused by the depletion of neurotransmitters from presynaptic terminals [9] (see Fig. 1). 


\subsection{Modeling synaptic facilitation-depression}

To describe the phenomenology described in the previous paragraph, we shall now recall how the membrane potential $V(x, t)$ of a single neuron $x$ at time $t$ is modeled. The derivation of the dynamics for $V(x, t)$ follows the steps described in classical theories of neural networks $[17,49,50]$ : The voltage potential $V(x, t)$ is driven by synaptic depression, described by a variable $\mu(t)$. This variable $\mu(t)$ characterizes the state of the synapse: the probability of vesicular release decreases following a train of stimulations. To account for this phenomena, after the arrival of an AP or a depolarization, vesicles are released, thus decreasing the total number and this change also decreases the probability of release of vesicles that should have been generated following an successive AP. For a single synapse, the dynamics of $\mu(t)$ is described by the phenomenological differential equation

$$
\dot{\mu}=\frac{1-\mu}{\tau}-U \mu F(V(t))
$$

where the first term in the r.h.s of eq. 1 is the rate of the vesicular replenishment, while the second one represents the rate of refilling, which is proportional to the fraction of available vesicles $\mu$ and the firing rate $F(V(t))$. The function $F$ is sigmoidal or a step function (Heaviside centered at a parameter $a$ ) of the membrane potential $V(t)$, see $[31,43]$.

Synaptic facilitation is a synaptic process by which a train of AP leads to an increase of vesicular release: the local biophysical description corresponds to the accumulation of residual calcium inside the synaptic cleft that results in increasing the vesicular release probability. Using the variable $x$ for the residual calcium, the empirical differential equation is

$$
\dot{x}=\frac{X-x}{t_{f}}+K(1-x) F(V(t))
$$

where $\mathrm{X}$ is the steady state level, $t_{f}$ is the relaxation time, which can be driven by diffusion and calcium exchangers, the second term in the r.h.s of 2 expresses that a train of stimulations increases $x$, which varies between $\mathrm{X}$ and $1 . K$ is a parameter that depends on the calcium influx.

\subsection{Synaptic connections and neuronal organization}

Neurons connect to each other through synapses. The distribution of synapses can be studied empirically by mapping the response recorded in neuron $y$ while stimulating neuron $x$. The recorded current amplitude represents the synaptic weight $w(x, y)$, proportional to the firing synchronization between neurons $x$ and $y$, (see Figs 5 and 7c in [4], for example). The synaptic weight can be represented by the generic family of functions

$$
w\left(\boldsymbol{x}_{n}, \boldsymbol{y}_{n}\right)=\frac{A}{B+\left|\boldsymbol{x}_{n}-\boldsymbol{y}_{n}\right|} \frac{1}{\sqrt{2 \pi} s} e^{-\frac{\left(\boldsymbol{x}_{n}-\boldsymbol{y}_{n}\right)^{2}}{2 s^{2}}},
$$

where $A, B, s$ are parameters that can fitted to data. By adjusting these parameters, various synaptic weight distribution of cortical organization can be accounted for. In the visual cortex, derivative of Gaussian (Mexican hat) are used [36].

The dynamics of a one dimensional connected ensemble of neurons in the interval $[0, L]$, driven by synaptic depression is modeled by

$$
(S)\left\{\begin{aligned}
\dot{V}(\boldsymbol{x}, t) & =-\alpha V(\boldsymbol{x}, t)+\int_{0}^{L} \mu(\boldsymbol{y}, t) w(\boldsymbol{x}, \boldsymbol{y}) F(V(\boldsymbol{y}, t)) d \boldsymbol{y}+\sigma \dot{\omega}(\boldsymbol{x}, t) \\
\dot{\mu}(\boldsymbol{x}, t) & =\frac{1-\mu(\boldsymbol{x}, t)}{\tau}-U \mu(\boldsymbol{x}, t) F(V(\boldsymbol{x}, t)), \text { for } \boldsymbol{x} \in[0, L] .
\end{aligned}\right.
$$

This system represents the dynamics of firing rate of an ensemble of neurons driven by synaptic depression and white noise $(\sigma \dot{\omega}(\boldsymbol{x}, t)$, uniform in $\boldsymbol{x}$ and Gaussian in time t). The continuum representation can lead to artificial 
difficulties that are not presented in discret neuronal network models. For a discret neural network, driven by synaptic depression and facilitation, the firing rate is given by the following system of equations $(\mathrm{i}=1 . . \mathrm{N})$,

$$
\begin{aligned}
\tau \dot{h_{i}} & =-h_{i}+\sum_{j} J_{i j} x_{j} y_{j} h_{j}^{+}+\sqrt{\tau} \sigma_{i} \dot{w}_{i}+H_{i} \delta_{k i} \delta\left(t-t_{s t i m}\right) \\
\dot{x}_{i} & =\frac{X_{j}-x_{i}}{t_{f}}+K_{j}\left(1-x_{i}\right) h_{i}^{+} \\
\dot{y}_{i} & =\frac{1-y_{i}}{t_{r}}-L_{j} x_{i} y_{i} h_{i}^{+},
\end{aligned}
$$

where $F(x)=x^{+}, J_{i j}$ is the mean number of connections (synapses) per neuron, $K_{i}$ and $L_{i}$ are chosen to be identical for all synapses of neuron $i$. We have included in the r.h.s of system eq. 4 a noise term that accounts for membrane fluctuation, which results from the homogenization of many independent vesicular releases. The last term accounts for a stimulation evoked at time $t_{\text {stim }}$ at neuron $k$ only. We have not described here models of voltage conductances for the neuronal intrinsic excitability. Interestingly, the synaptic dynamics presented in systems 4 and 4 is sufficiently rich to capture many network dynamics, such as epileptic activity, wave spreading [4,6], bursting [14], Up- and Down- states (described below) [23]. Interestingly, contrary to detailed models where all neurons are considered, the ones presented here are trackable analytically.

\subsection{Synaptic facilitation-depression modeling of neuronal network}

For sufficiently connected neuronal network, when all neurons inside behave identically, the ensemble can be characterized by the average firing rate $h$, an averaged facilitation parameter $x$ and the averaged depression $y$ [31]. Of course, this dynamics cannot be generated by intrinsic properties of a single neuron. A mean-field model describing a sufficiently connected neuronal network with an averaged number of synapses per neurons and synaptic dynamics is the one of a single homogeneous excitatory neuronal population. The mean-field system of equation derived from eq. 4 is

$$
\begin{aligned}
\tau \dot{h} & =-h+J x y h^{+}+\tau H \delta\left(t-t_{\text {stim }}\right)+\sqrt{\tau} \sigma \dot{w} \\
\dot{x} & =\frac{X-x}{t_{f}}+K(1-x) h^{+} \\
\dot{y} & =\frac{1-y}{t_{r}}-L x y h^{+},
\end{aligned}
$$

where an experimental spike stimulation is accounted for by the term $H \delta\left(t-t_{\text {stim }}\right)$, which sets at time $t_{\text {stim }}$ the average firing rate of the network to the value $H$. The average population firing rate $R=h^{+}=\max (h, 0)$ is a threshold linear function of synaptic current. The term Jxy reflects the combined effect of synaptic short-term dynamics and network activity. The second equation describes the mean facilitation dynamics, which enters into a depression process described in the third equation [31]. $J$ measures the mean number of connections (synapses) per neuron, while $K$ and $L$ account for the mean biophysical characteristics by which the firing rate is transformed into molecular events, changing the duration and the probability for vesicular release respectively. More precisely, in a biophysical context, $L$ is the rate at which vesicles are depleted for a given firing rate and $\mathrm{K}$ measures the rate of facilitation. The derivation of system eq. 5 can be obtained by averaging system eq. 4 (see [43] and [7]). In general, the parameters $t_{r}, t_{f}, \tau L, K$ are obtained from comparing the response of the model with experimental data [13].

\section{Description of BURsting ReVERBERAtion}

Few seconds bursting induced experimentally by stimulating a single neuron is generated in an ensemble of neurons. The burst duration can be several seconds (Fig. 2). In that example, all neurons behave identically [9]. 

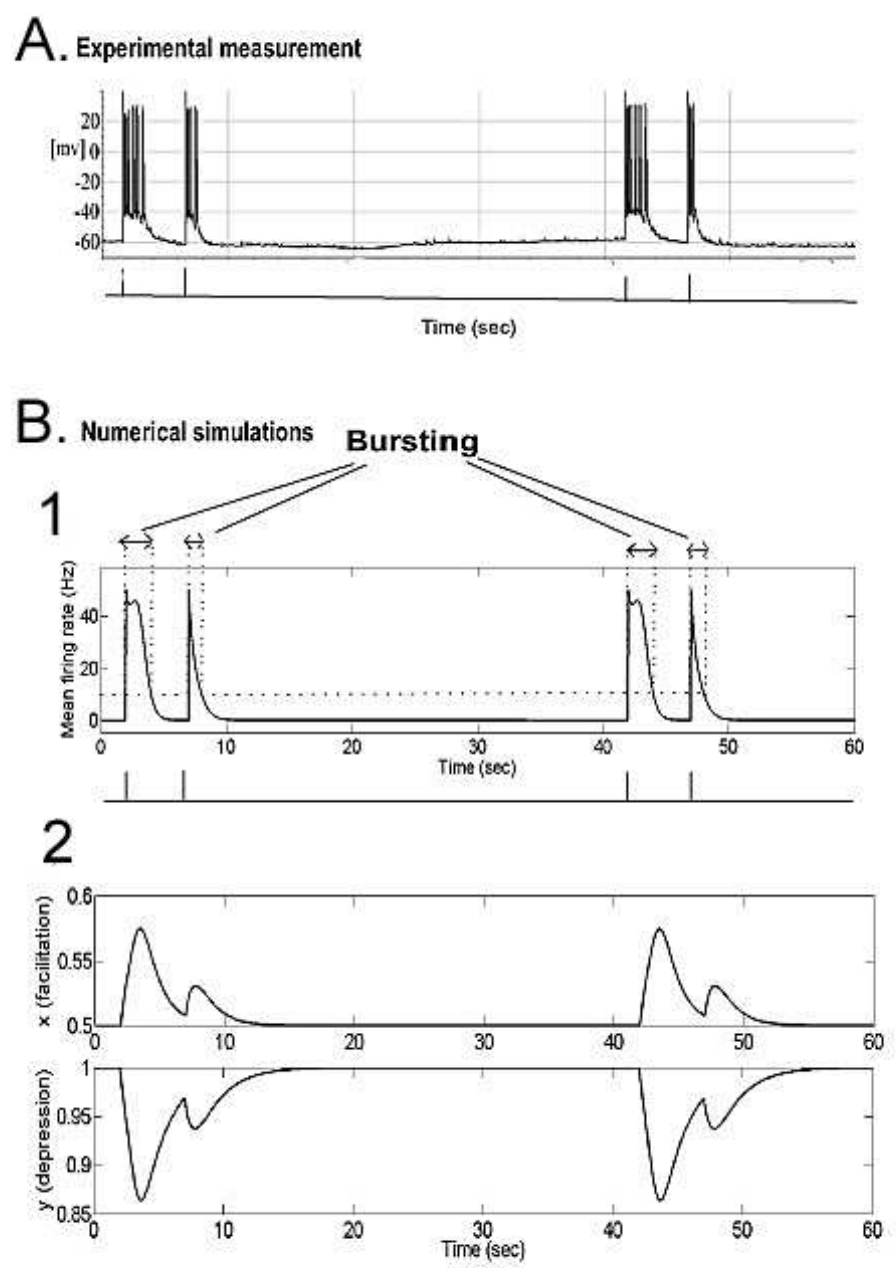

FIGURE 2. Synaptic depression-facilitation model accounting for bursting reverberation. (A) Evoked bursts generated in a microculture hippocampal neurons [10], with a single action potential evoked alternately at 5 and 35 seconds intervals, showing a reduction in burst duration when the bursts are generated at short intervals. (B) Following the experimental protocol, we simulated with equations 5 and parameters of Table 1 , the mean firing rate response. The corresponding facilitation and depression variables show the level of neuronal network activity underlying the overall dynamics. $(C)$ Plot of the product $x y$ of facilitation by the depression variable, which represents for the total synaptic activity. (D) Burst durations at 35 seconds and 5 seconds intervals. We compare the burst durations from experimental data $(\mathrm{n}=20)$ with numerical simulations of equations 5.

We have shown in [25] that the mean-field modeling using tuned parameters can reproduce the neuronal network dynamics. In combination with previous experimental data that have rules out other mechanisms responsible 
for bursting, it was shown that bursting can be described by depression-facilitation model as presented in section 1.3 .

\subsection{Definition of the reverberation time}

Because the burst duration depends strongly on the synaptic properties, the effect of changing the total synaptic connections (variable J) can be studied by estimating analytically the reverberation time for a well connected network. Following the experimental protocol, in a simulation, an induced spike at time $t=0$ sets the firing rate $h$ to a value $H$ and the reverberation time $T_{R}$ is defined as the first time when the average firing rate $h$ reaches the threshold value $h_{T}=10 \mathrm{~Hz}$ under which we consider that there is no more synchronous bursting activity. Thus, we define the reverberation time as a first hitting time

$$
T_{R}=\inf \left\{t>0, h(t)=h_{T}\right\}
$$

To estimate $T_{R}$, we have approximated during the early bursting period the firing rate by $h(t) \approx H$ in the last two equations of system (5) [13]. Although this approximation can affect drastically the depression $x$ and facilitation $y$ variable, later on in the decay phase of $h$, it does not impact the behavior of the average firing rate $h$, which depends on $x$ and $y$ essentially in the initial phase. In general, there is no precise theory to study the time for long trajectories to end up at a given point. The local properties of the dynamics are used here to obtain the first approximation term [13]

$$
h(t)=H \exp \left(-\frac{t}{\tau}+J \int_{0}^{t} x(s) y(s) d s\right) .
$$

Integral $\int_{0}^{t} x(s) y(s) d s$ is estimated in terms of the variable $-t_{d} L H X\left(1+\frac{K H}{1 / t_{f}+K H}\right)$. Solving the equation $h\left(T_{R}\right)=h_{T}$, we obtained that

$$
\begin{aligned}
T_{R}(J) & =\frac{\theta-\sqrt{\theta^{2}-2 J \tau X H(K-L X) \tau \ln \left(\frac{H}{h_{t h}}\right)}}{J \tau X H(K-L X)} \\
\theta & =1-J X \tau .
\end{aligned}
$$

showing that the reverberation $T_{R}$ is a sub-linear function of the synaptic connectivity $J$ [13]. Thus as the mean synaptic wight $J$ increases, the reverberation time starts increasing slowly, confirming that many synapses have to be formed before the reverberation time starts changing. A possible interpretation is that learning is strongly nonlinear: while it starts slowly, it effect is getting stronger after several synapses are already formed. Numerical simulations of system eq. 5 show that the reverberation time has a bell shaped relation with J. Before reaching its maximum, we showed above that this reverberation time increases sub-linearly, however, the analysis of the maximum and the decreasing phase is an open question that should be estimated from the dynamical system eqs. 5. A preliminary conclusion is that synaptic intrinsic properties and network connectivity shape the mean burst duration, which persists across various network scales (system eq. 5 is independent of the number of synapses or neurons).

\section{Modeling the SPontaneous aCtivity of Neuronal Networks: Up- And DOWN-STATES DYNAMICS}

It remains intriguing that the cerebral cortex is continuously active, even in the absence of external stimuli, showing patterns of activity that resemble the ones generated by direct stimulation [28]. Recurrent patterns have also been found in neuronal ensembles [39]. Yet the origin of these recurrent activities based on network properties remains unexplained. Recording the spontaneous activity connected neurons has revealed that the 
membrane potential of neurons can switch between two different values called Up- and Down-states [2,8,11,39]. We review here our effort to model these Up- and Down- state transitions based on synaptic-depression and using mean-field equations [44]. The mean neuronal activity can be described by mean-field model eq. 5 with synaptic depression only, leading to the reduced stochastic equations $[4,23]$

$$
\begin{aligned}
\tau \dot{V} & =-V+J \mu R(V)+\sqrt{\tau} \sigma \dot{\omega} \\
\dot{\mu} & =\frac{1-\mu}{t_{r}}-U \mu R(V),
\end{aligned}
$$

where $V$ is an average voltage (measured in $\mathrm{mV}$ with a base line at $0 \mathrm{mV}$ ), $J$ is the average synaptic strength in the network, $U$ and $t_{r}$ are two parameters $[4,44] . \dot{\omega}$ is the $\delta$-correlated white noise of mean zero and variance one. $R(V)$ is the average firing rate (in $\mathrm{Hz}$ ) approximated by a threshold-linear voltage dependence function

$$
R(V)= \begin{cases}\alpha(V-T) & \text { if } V>T \\ 0 & \text { else. }\end{cases}
$$

where $T>0$ is a threshold and $\alpha=1 H z / m V$ is a conversion factor. As discussed in the previous sections, the function $R(V)$ can be sigmoidal to account for the saturation of the neuronal firing rate. However, due to the synaptic-depression, the dynamics never reaches the saturation regime and thus we can use the function $R(V)$ defined above. An alternative possibility for system 9 is to consider the case where a noise term is added in the second equation, however results are almost identical.
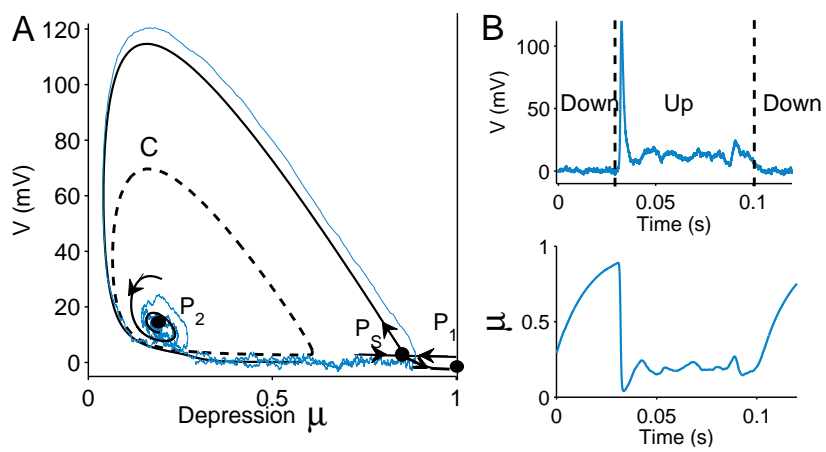

Figure 3. Transition to an Up-state. (A): Phase portrait $(V, \mu)$ defined by Eq. 9, containing a stochastic trajectory (blue). The phase space shows a limit cycle $\mathrm{C}$ (dashed line) containing a stable focus $P_{2}$, a saddle point $P_{S}$ and a stable attractor $P_{1}$. The Up-state is the domain inside the limit cycle C. $(\mathbf{B})$ : transition of a trajectory to an Up-state in the time domain for the variable $\mathrm{V}$ and $\mu$.

\subsection{Phase-space analysis of the Up- and Down- state model}

The phase-space associated with the deterministic part of the dynamical system 9 is defined by the variables $(V, \mu)$ (see Fig. 3A). When the parameter $\mathrm{J}$ is large enough [23], the phase space contains three critical points: two attractors $P_{1}$ (located at $\left.V=0, \mu=1\right), P_{2}$ and one saddle point $P_{S}$. The basin of attraction of the stable focus $P_{2}$ is delimited by the unstable limit cycle $C$ (dashed line in Fig. 3A). This basin defines the Up-state region in the modeling. This basin of attraction emerges when the network connectivity $J$ exceeds a minimal value (see [23]). As the parameter $J$ increases, following a super-critical (with a minus sign) Hopf-bifurcation, 
the point $P_{2}$, which was previously a repulser becomes an attractor and an unstable limit cycle appears around it (Fig. 2A). Intuitively, this bifurcation occurs when the recurrent connections are becoming sufficiently strong. Simulations of the stochastic eq. 9 show trajectories entering in the limit cycle by a noise induced transition (see Fig.3B). The stable focus is at the intersection of nullclines. The residence time in the Up-state is precisely the mean first passage time spent by a stochastic trajectory before it reaches the unstable limit cycle (dashed line). Once a trajectory exits through the limit cycle, the dynamic relaxes exponentially to the Down-state (not shown here).

An open question in the field of neuroscience is to understand what defines the duration of the Up-states and their oscillation frequencies. The interspike interval (time between two consecutive population spikes) corresponds to the time for a trajectory to go around the attractor of the Up-state. Entering into an Up-state is a succession of crossing separatrices and entering into the unstable limit cycle. It is known that entering into an Up-state is associated with a fast rotation (population spike) around the unstable limit cycle and a small noise fluctuation can produce the impulsion necessary to create a transition to an Up-state [23]. As we shall see, the oscillation frequencies in Up-state is simply given by the imaginary part of the eigenvalue at the focus, which can be resolve by a classical analysis of the deterministic part of system eqs. 5 . However, the distribution of times spent in Up-states (DTUS) can be studied using the residence time in the basin of attraction of the stable focus $P_{2}$ described above [25], but it requires accounting for the local and global structure of the basin of attraction. The phase space geometry is quite unusual as the stable focus is located very close to the unstable limit cycle, leading to novel and dynamics that has not been previously much discussed (see also [48]). Using numerical simulations, we found that for a specific range of the noise amplitude, the distribution of times in the Up-state shows some peaks Fig. 6, which are due to winding around the focus, before escaping from the basin of attraction. Thus the time in the Up-state does not follow a Poissonian law. We propose now to clarify these issues.

\subsection{An unusual activation escape from the Up-state}

The mean time spent by a stochastic trajectory in an attractor is the reciprocal of the escape rate. It has been computed based on asymptotic analysis of elliptic PDE and Large Deviation Principle [19,34,46]. However this analysis is not sufficient to characterize the escape when the focus is located close the separatrix as it is the case here. Indeed, the DTUS shows various oscillation peaks in Fig. 6, confirming that it is non-Poissonian and in addition, the amplitude of the DTUS depends on the noise size but not the frequency.

For a focus located near the limit cycle, the vector field vanishes close to the boundary, leading to a dynamics dominated by the noise. Thus escape should occur in a small region of the limit cycle close to the focus. Consequently, if a trajectory does not exit in that small region, it winds around the focus before returning to the region where it can escape. This is shown in Fig. 4. This rotation phenomena is responsible for the peaks in the DTUS. Moreover, the periodic peaks in DTUS are associated with a distribution of exit points concentrated in a very small part of the limit cycle, as shown in Fig. 6.

We conclude at this stage that these peaks are a direct consequence of the dynamics induced by the geometrical organization of the recurrent ensembles, where the focus is very close to the unstable limit cycle. As the noise decreases, the distribution of exit time evolves from an initially decaying exponential to an oscillatory decay with several apparent peaks. Thus the residence time of the membrane potential in the cortical Up-state does not follow a Poissonian law, but presents several peaks depending on the parameter values. Contrary to what we though earlier, these peaks are neither artifacts of numerical simulations nor electrophysiological measurements, but are inherent properties of the underlying synaptic dynamics. We will now investigate the stochastic nature of these peak oscillations. 

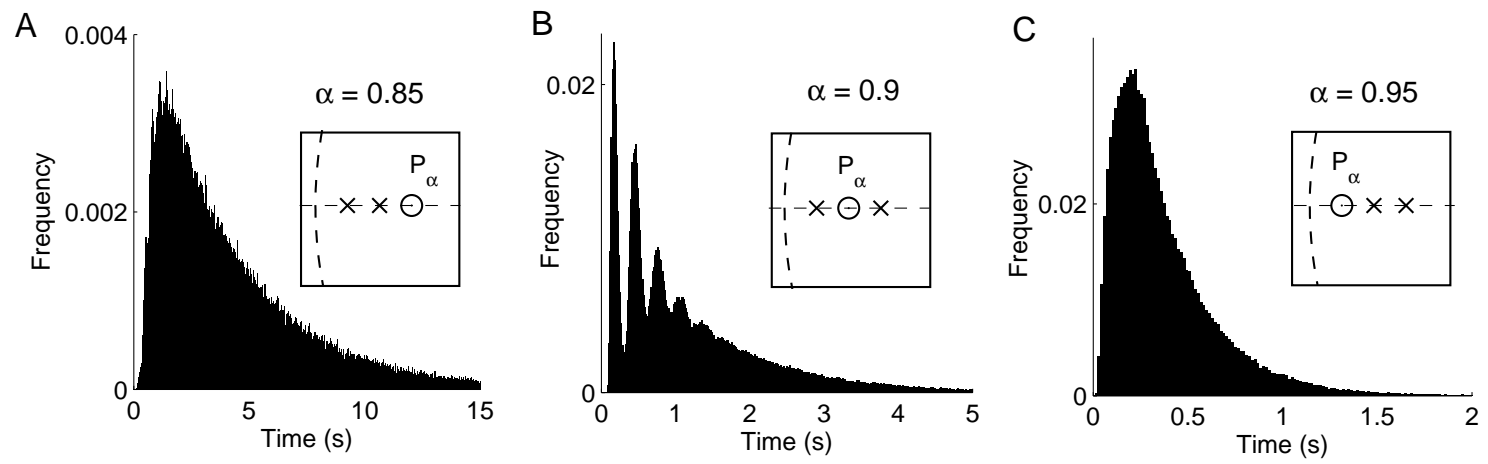

Figure 4. Peak resonances occur when the stable point is at an optimal distance from the limit cycle. Histograms of exit time for different values of $\alpha=0.95$ (a), 0.9 (b) and 0.85 (c) (number of runs is 250000, starting point $=(-0.8,0)$ ). When the stable point is far away from the limit cycle (left), the peaks are disappearing in the distribution tails. As the focus approaches the boundary, peaks appear (middle), until all trajectories concentrate in the first peak (right), when the distance becomes too close.

\section{Generic stochastic model for Up- And Down- States: Analysis And SIMULATIONS OF THE EXIT TIME FROM AN ASYMMETRIC FOCUS TO A LIMIT CYCLE}

To clarify the origin of the peak oscillations in the escape problem, we present now an escape problem from an attractor, where the field $\boldsymbol{b}$ has a single focus point $\mathrm{A}$ in $\Omega(\boldsymbol{b}(A)=0)$ close to the boundary of the the two-dimensional domain $\Omega$. We use the following Hopf-class of systems defined inside the unit disk $D(1)$ :

$$
\dot{z}=\boldsymbol{b}_{0}(z)=\lambda z\left(-1+|z|^{2}+i \omega\right),
$$

where $\lambda>0$ and $\omega \in \mathbb{R}$ (now on, we choose $\lambda=1$ ). The field $\boldsymbol{b}_{0}$ has an attractor at the center and the circle $|z|=1$ is an unstable limit cycle. This is the classical super-critical normal form of the Hopf-system in the complex coordinates $z=x+i y$ where $\mathrm{x}, \mathrm{y}$ are real. To obtain a dynamical system with an attractor arbitrarily close to the limit cycle, we conjugate the field with the Mobius transformation

$$
\Phi_{\alpha}(z)=\frac{z-\alpha}{1-\alpha z}, \quad 0<\alpha<1
$$

where $-\alpha \in]-1,0]$. We obtain a one parameter vector-field $\boldsymbol{b}_{\alpha}$ conjugated to $\boldsymbol{b}_{0}$ by the mapping $\Phi_{\alpha}$. The Mobius transformation sends the center 0 to the point $P_{\alpha}=-\alpha$, which can be arbitrarily close to the boundary point $z=-1$. Moreover, the characteristic boundary $|z|=1$ is preserved. Details of the analysis are provided in [15]. The expression of the field is

$$
\begin{aligned}
\boldsymbol{b}_{\alpha}(z) & =\Phi_{\alpha}^{\prime}\left(\Phi_{\alpha}^{-1}(z)\right) \boldsymbol{b}_{0}\left(\Phi_{\alpha}^{-1}(z)\right) \\
& =\lambda \frac{(z+\alpha)(1+\alpha z)}{\left(1-\alpha^{2}\right)}\left(-1+\left|\frac{z+\alpha}{1+\alpha z}\right|^{2}+i \omega\right) .
\end{aligned}
$$

The vector field can be decomposed near the boundary as

$$
\boldsymbol{b}_{\alpha}(\rho, \theta)=-\rho\left[b_{\alpha}^{0}(\theta)+O\left(\rho^{2}\right)\right] \boldsymbol{\nu}+b_{\alpha}^{*}(\rho, \theta) \boldsymbol{t},
$$


where $\boldsymbol{t}$ and $\boldsymbol{\nu}$ are the unit tangent and outer-normal respectively at the boundary $\partial D$ at $(0, \theta)$. $\rho$ is the distance to the origin and $\theta$ the polar angle. The components $b_{\alpha}^{0}(\theta)$ and $b_{\alpha}^{*}(\rho, \theta)$ near the limit cycle are given by

$$
\begin{aligned}
b_{\alpha}^{0}(\theta) & =\frac{2\left(1-\alpha^{2}-\omega \alpha \sin (\theta)\right)}{1-\alpha^{2}}+O(\rho), \\
b_{\alpha}^{*}(\theta)=b_{\alpha}^{*}(0, \theta) & =\frac{\omega}{1-\alpha^{2}}\left(1+2 \alpha \cos (\theta)+\alpha^{2}\right)+O(\rho) .
\end{aligned}
$$
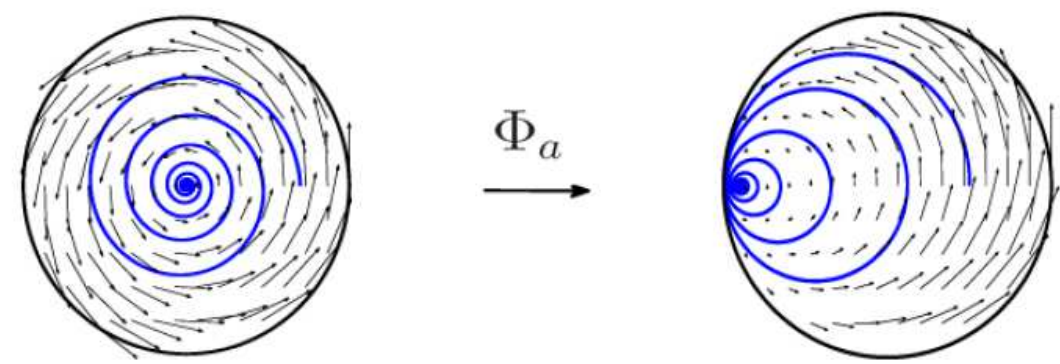

FIGURE 5. Mobius mapping of the Hopf vector-field. The mapped generic vector field has a focus arbitrarily close to the unstable limit cycle. Hopf vector-field (Left) and its conjugated (Right).

\subsection{Distribution of exit points}

We now study the escape from the unit disk $D$ for the stochastic process

$$
d \boldsymbol{x}_{\varepsilon}(t)=\boldsymbol{b}_{\alpha}\left(\boldsymbol{x}_{\varepsilon}(t)\right) d t+\sqrt{2 \varepsilon} d \boldsymbol{w}(t)
$$

where $\varepsilon$ is a small parameter. The probability density $P_{\alpha}(\theta)=\operatorname{Pr}\left\{\boldsymbol{x}_{\varepsilon}(\tau)=\theta\right\}$ of exit points defined on the boundary $\partial D$ is determined by the $2 \pi$-periodic solution $\xi_{\alpha}(s)$ of the Bernoulli equation the form

$$
-\sigma(\theta) \xi_{\alpha}^{3}(\theta)+\left[b_{\alpha}^{0}(\theta)\right] \xi_{\alpha}(\theta)+b_{\alpha}^{*}(\theta) \xi_{\alpha}^{\prime}(\theta)=0,
$$

where $\sigma(\theta)=\boldsymbol{\sigma}(0, \theta) \boldsymbol{\nu}(0, \theta) \cdot \boldsymbol{\nu}(0, \theta)$. The pdf $P_{\alpha}(\theta)$ is given by [34], [46, eq.(10.127)]

$$
P_{\alpha}(\theta)=\frac{\frac{\xi_{\alpha}^{2}(\theta) \sigma(\theta)}{b_{\alpha}^{*}(0, \theta)}}{\int_{0}^{2 \pi} \frac{\xi_{\alpha}^{2}(s) \sigma(s)}{b_{\alpha}^{*}(0, s)} d s} .
$$

After using expressions 13 and the solution of equation 15, we obtain the explicit expression for the density of exit points ( $\varepsilon$ is fixed but small and alpha can vary in small interval away of 1) [15]

$$
P_{\alpha}(\theta)=\frac{\left(1+2 \alpha \cos \theta+\alpha^{2}\right)^{-3}}{\int_{-\pi}^{\pi}\left(1+2 \alpha \cos s+\alpha^{2}\right)^{-3} d s} .
$$

As $\alpha \rightarrow 1$, the density $P_{\alpha}(\theta)$ concentrates at the point $\theta=\pi$ on the boundary (Fig.6). Brownian simulations and the asymptotic expression 17 show that the distribution of exit points on $\partial D$ is almost zero, except for a 
small interval $R(\alpha)$ centered at the boundary point, closest to focus (see also [25] for details). This result shows that exit occurs only in a small arc of the boundary and when a trajectory fails to hit the boundary, while being in the neighborhood of this arc, it has to wind around the focus and return to the region $R(\alpha)$. Stochastic simulations of eq. 14 shows that the history of each trajectory and allow to evaluate the contribution of each winding to the exit time distribution by monitoring the winding number prior to exit. Thus the residence time of trajectories before exit is quantized by the winding numbers around the focus, as confirmed in the empirical statistics presented in Fig.7.
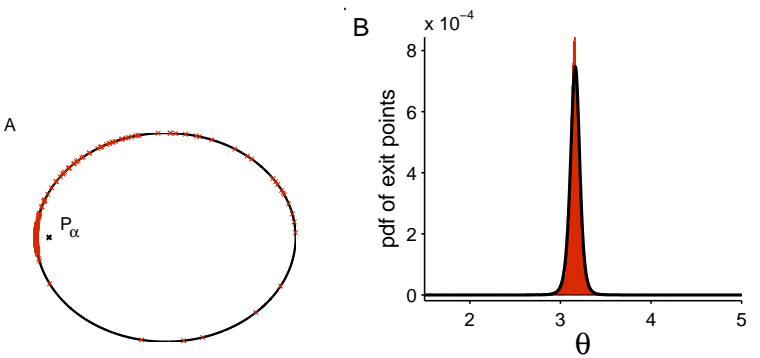

Figure 6. Density of exit points.(Left): Exit points are marked red. (Right) Histogram of exit points (red) and the density $P_{\alpha}(\theta)$ from (17) (black). Simulation parameters are $\alpha=$ $-0.9, \omega=10, \varepsilon=0.005$. Number of runs $=50000$.
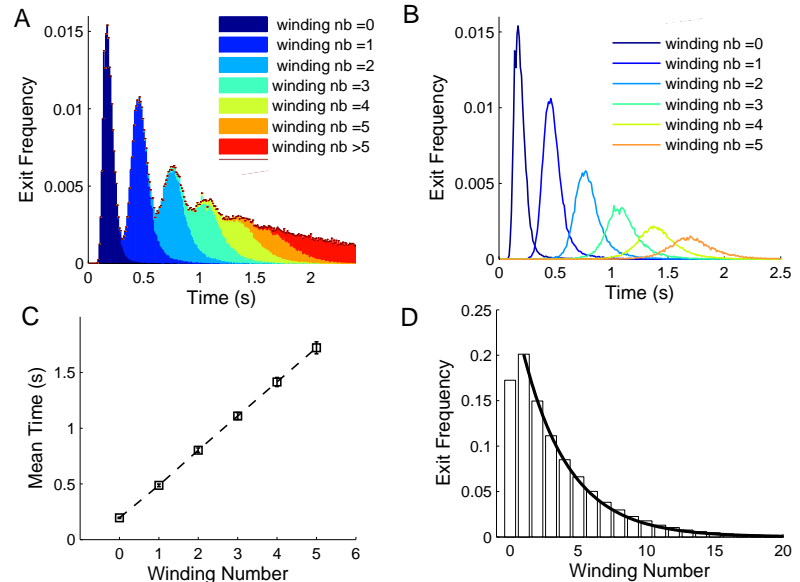

FIGURE 7. Statistic of exit trajectories and winding. A: The different colors mark the exit frequencies for different winding numbers. B: Exit frequencies conditioned on winding number (0 to 5 turns). C: Mean of exit times in histogram B versus the winding number. D: Histogram of winding numbers in $\mathbf{A}$. The exponential function $f(n)=p(1-p)^{n-1}$ (solid line) approximates the decay rate. The ratio $p$ of frequencies of the first to the second turn is the probability to exit without making a turn. 


\subsection{Characterizing the exit time distribution by solving asymptotically the Fokker-Planck Equation}

The survival probability density function (pdf) of the process 34 before absorbtion satisfies the Fokker-Planck equation (FPE)

$$
\begin{aligned}
& \frac{\partial p(\boldsymbol{y}, t \mid \boldsymbol{x})}{\partial t}=L \boldsymbol{y} p(\boldsymbol{y}, t \mid \boldsymbol{x}) \text { for } \boldsymbol{x}, \boldsymbol{y} \in D \\
& p(\boldsymbol{y}, t \mid \boldsymbol{x})=0 \text { for } \boldsymbol{x} \in \partial D, \boldsymbol{y} \in D, t>0 \\
& p(\boldsymbol{y}, 0 \mid \boldsymbol{x})=\delta(\boldsymbol{y}-\boldsymbol{x}) \text { for } \boldsymbol{x}, \boldsymbol{y} \in D
\end{aligned}
$$

where $\boldsymbol{\sigma}(\boldsymbol{x})=\boldsymbol{b}(\boldsymbol{x}) \boldsymbol{b}^{T}(\boldsymbol{x})=\boldsymbol{I} \boldsymbol{d}$. The Fokker-Planck operator $L_{\boldsymbol{y}}$ is defined by

$$
L \boldsymbol{y} u(\boldsymbol{y})=\varepsilon \sum_{i, j=1}^{2} \frac{\partial^{2}\left[\sigma^{i, j}(\boldsymbol{y}) u(\boldsymbol{y})\right]}{\partial y^{i} \partial y^{j}}-\sum_{i=1}^{2} \frac{\partial\left[a^{i}(\boldsymbol{y}) u(\boldsymbol{y})\right]}{\partial y^{i}}
$$

and its adjoint is

$$
L_{\boldsymbol{x}}^{*} v(\boldsymbol{x})=\varepsilon \sum_{i, j=1}^{2} \sigma^{i, j}(\boldsymbol{x}) \frac{\partial^{2} v(\boldsymbol{x})}{\partial x^{i} \partial x^{j}}+\sum_{i=1}^{2} a^{i}(\boldsymbol{x}) \frac{\partial v(\boldsymbol{x})}{\partial x^{i}}
$$

The non-self-adjoint operators $L_{\boldsymbol{y}}$ and $L_{\boldsymbol{x}}$ with homogeneous Dirichlet boundary conditions have the same eigenvalues $\lambda_{n, m}$, because the equations are real and the eigenfunctions $u_{n, m}(\boldsymbol{y})$ of $L_{\boldsymbol{y}}$ and $v_{n, m}(\boldsymbol{x})$ of $L_{\boldsymbol{x}}^{*}$ are bases that are bi-orthonormal in the complex Hilbert space such that

$$
\int_{D} \bar{v}_{n, m}(\boldsymbol{y}) L_{\boldsymbol{y}} u_{n, m}(\boldsymbol{y}) d \boldsymbol{y}=\int_{D} \bar{u}_{n, m}(\boldsymbol{y}) L_{\boldsymbol{y}}^{*} v_{n, m}(\boldsymbol{y}) d \boldsymbol{y}=\delta_{n, m}
$$

The solution of the FPE can be expanded as

$$
p_{\varepsilon}(\boldsymbol{y}, t \mid \boldsymbol{x})=e^{-\lambda_{0} t} u_{0}(\boldsymbol{y}) v_{0}(\boldsymbol{x})+\sum_{n, m} e^{-\lambda_{n, m} t} u_{n, m}(\boldsymbol{y}) \bar{v}_{n, m}(\boldsymbol{x})
$$

where $\lambda_{0}$ is the real-valued principal eigenvalue and $u_{0}, v_{0}$ are the corresponding positive eigenfunctions, that is, solutions of $L \boldsymbol{x}\left(u_{0}\right)=-\lambda_{0} u_{0}$ and $L_{\boldsymbol{y}}^{*}\left(v_{0}\right)=-\lambda_{0} v_{0}$, respectively.

\subsection{The spectrum of the nonself-adjoint Fokker-Planck operator}

To demonstrate the oscillatory decay of the survival probability of the stochastic dynamics $d \boldsymbol{x}_{\varepsilon}=\boldsymbol{a}\left(\boldsymbol{x}_{\varepsilon}\right) d t+$ $\sqrt{2 \varepsilon} \boldsymbol{b}\left(\boldsymbol{x}_{\varepsilon}\right) d \boldsymbol{w}$, we study its activation by small noise over the boundary of the domain of attraction $D$ of a stable focus. We compute the leading-order asymptotic expansion of all eigenvalues $\lambda_{n, m}$ of the non-self-adjoint operators $L_{\boldsymbol{y}}$. Using asymptotic analysis, we obtained [24] the asymptotic structure of the eigenfunctions of $L_{\varepsilon}$ and of its adjoint $L_{\varepsilon}^{*}$ for small $\varepsilon$ and the higher-order eigenvalues. They are given by

$$
\lambda_{m, n}=n \omega_{1}+m i \omega_{2}+O(\varepsilon)
$$


for $n=1,2, \ldots, m= \pm 1, \ldots$, where the frequencies $\omega_{1}$ and $\omega_{2}$ are defined as

$$
\omega_{1}=\frac{\omega_{2}}{2 \pi} \int_{0}^{S} \frac{\sigma(s) \xi^{2}(s)}{B(s)} d s \text { and } \omega_{2}=\frac{2 \pi}{\int_{0}^{S} \frac{d s}{B(s)}}
$$

where we have used the following notation: $s$ is arclength on $\partial D=\{\boldsymbol{x}(s): 0 \leq s<S\}$, measured clockwise, $\boldsymbol{n}(\boldsymbol{x})$ is the unit outer normal at $\boldsymbol{x} \in \partial D, B(s)=\left|\boldsymbol{b}_{\boldsymbol{\alpha}}(\boldsymbol{x}(s))\right|, \boldsymbol{x}(s)$ parametrizes the limit cycle and $\sigma(s)=$ $\boldsymbol{n}(\boldsymbol{x}(s))^{T} \boldsymbol{\sigma}(\boldsymbol{x}(s)) \boldsymbol{n}(\boldsymbol{x}(s))$.

\subsection{Method for computing the spectrum of $L y$}

To compute the spectrum of $L \boldsymbol{y}$, we use matched asymptotics [5] to construct a uniform asymptotic approximation to the eigenfunctions $u_{m, n}(\boldsymbol{y})$. We begin with the outer expansion of the eigenfunction $u(\boldsymbol{y})$ in the WKB form

$$
u(\boldsymbol{y})=K_{\varepsilon}(\boldsymbol{y}) \exp \left\{-\frac{\psi(\boldsymbol{y})}{\varepsilon}\right\}
$$

where the function $\psi(\boldsymbol{y})$ is a solution of eikonal equation

$$
\boldsymbol{\sigma}(\boldsymbol{y}) \nabla \psi(\boldsymbol{y}) \cdot \nabla \psi(\boldsymbol{y})+\boldsymbol{a}(\boldsymbol{y}) \cdot \nabla \psi(\boldsymbol{y})=0
$$

(see [46, Chap.10]) and $\psi(\boldsymbol{y})$ is constant on the boundary. The function $K_{\varepsilon}(\boldsymbol{y})$ is a regular function of $\varepsilon$ for $\boldsymbol{y} \in D$, but has to develop a boundary layer to satisfy the homogenous Dirichlet boundary condition $K_{\varepsilon}(\boldsymbol{y})=0$ for $\boldsymbol{y} \in \partial D$. Therefore $K_{\varepsilon}(\boldsymbol{y})$ is further decomposed into the product

$$
K_{\varepsilon}(\boldsymbol{y})=\left[K_{0}(\boldsymbol{y})+\varepsilon K_{1}(\boldsymbol{y})+\cdots\right] q_{\varepsilon}(\boldsymbol{y}),
$$

where $K_{0}(\boldsymbol{y}), K_{1}(\boldsymbol{y}), \ldots$ are regular functions in $D$ and on its boundary and are independent of $\varepsilon$, and $q_{\varepsilon}(\boldsymbol{y})$ is a boundary layer function. The boundary layer function $q_{\varepsilon}(\boldsymbol{y})$ satisfies the boundary condition $q_{\varepsilon}(\boldsymbol{y})=0$ for $\boldsymbol{y} \in$ $\partial D$, the matching condition $\lim _{\varepsilon \rightarrow 0} q_{\varepsilon}(\boldsymbol{y})=1$ for all $\boldsymbol{y} \in D$. To find the boundary layer equation, we introduce the stretched variable $\zeta=\rho / \sqrt{\varepsilon}$ and define $q_{\varepsilon}(\boldsymbol{x})=Q(\zeta, s, \varepsilon)$. Expanding all functions in (24) in powers of $\varepsilon$ we find

$$
Q(\zeta, s, \varepsilon) \sim Q^{0}(\zeta, s)+\sqrt{\varepsilon} Q^{1}(\zeta, s)+\cdots
$$

and we obtain the boundary layer equation

$$
\sigma(s) \frac{\partial^{2} Q^{0}(\zeta, s)}{\partial \zeta^{2}}-\zeta\left[b^{0}(s)+2 \sigma(s) \phi(s)\right] \frac{\partial Q^{0}(\zeta, s)}{\partial \zeta}-B(s) \frac{\partial Q^{0}(\zeta, s)}{\partial s}=0
$$

where $\sigma(s)=(\sigma(0, s) \boldsymbol{n} \cdot \boldsymbol{n})$. The solution that satisfies the boundary and matching conditions $Q^{0}(0, s)=$ $0, \quad \lim _{\zeta \rightarrow-\infty} Q^{0}(\zeta, s)=1$ is given by

$$
Q^{0}(\zeta, s)=-\sqrt{\frac{2}{\pi}} \int_{0}^{\xi(s) \zeta} e^{-z^{2} / 2} d z
$$

where $\xi(s)$ is the $S$-periodic solution of the Bernoulli equation

$$
\sigma(s) \xi^{3}(s)+\left[b^{0}(s)+2 \sigma(s) \phi(s)\right] \xi(s)+B(s) \xi^{\prime}(s)=0 .
$$


The asymptotic expansion of higher-order eigenfunctions is constructed by the method used above to derive that of the principal eigenfunctions. First, we consider higher-order eigenfunctions of the adjoint problem, which leads to the boundary layer equation and matching conditions

$$
\begin{gathered}
\frac{\partial^{2} \tilde{Q}^{0}(\eta, s)}{\partial \eta^{2}}+\eta \frac{\partial \tilde{Q}^{0}(\eta, s)}{\partial \eta}+\frac{B(s)}{\sigma(s) \xi^{2}(s)} \frac{\partial \tilde{Q}^{0}(\eta, s)}{\partial s}=-\frac{\lambda}{\sigma(s) \xi^{2}(s)} \tilde{Q}^{0}(\eta, s) \\
\tilde{Q}^{0}(0, s)=0, \quad \lim _{\eta \rightarrow-\infty} \tilde{Q}^{0}(\eta, s)=0 .
\end{gathered}
$$

Applying the method of separating variable $\tilde{Q}^{0}(\eta, s)=R(\eta) T(s)$, we obtain $R_{n}(\eta)=\exp \left\{-\frac{\eta^{2}}{2}\right\} H_{2 n+1}\left(\frac{\eta}{\sqrt{2}}\right)$, where $H_{2 n+1}(x)$ are the Hermite polynomials of odd orders [1]. The function $T(s)$ is given by

$$
T(s)=\exp \left\{-\lambda \int_{0}^{s} \frac{d s^{\prime}}{B\left(s^{\prime}\right)}+2 n \int_{0}^{s} \frac{\sigma\left(s^{\prime}\right) \xi^{2}\left(s^{\prime}\right)}{B\left(s^{\prime}\right)} d s^{\prime}\right\} .
$$

The details are given in [24].

\subsection{When is the Mean first Passage Time (MFPT) of order 1?}

To evaluate the MFPT for the stochastic escape of system 14, we use the asymptotic formula [34]

$$
\bar{\tau}(\mathbf{0}) \sim \frac{\pi^{3 / 2} \sqrt{2 \varepsilon \operatorname{det} \boldsymbol{Q}}}{\int_{0}^{S} K_{0}(s) \xi(s) d s} \exp \left\{\frac{\hat{\psi}}{\varepsilon}\right\}
$$

where $\hat{\psi}$ is a constant, the function $K_{0}(s)$ is given by

$$
K_{0}(s)=\frac{1}{B(s)} \exp \left\{-\int_{0}^{s}\left[\frac{a_{0}\left(s^{\prime}\right)-\xi^{2}\left(s^{\prime}\right)}{B\left(s^{\prime}\right)} d s^{\prime}\right]\right\},
$$

and $\xi(s)$ is the $S$-periodic solution of the Bernoulli equation

$$
\sigma(s) \xi^{3}(s)+\left[a^{0}(s)+2 \sigma(s) \phi(s)\right] \xi(s)+B(s) \xi^{\prime}(s)=0 .
$$

The solution $\psi(\boldsymbol{x})$ of the Eikonal equation (25) near the origin (the focus) is given by

$$
\psi(\boldsymbol{x})=\frac{1}{2} \boldsymbol{x}^{T} \boldsymbol{Q} \boldsymbol{x}+o\left(|\boldsymbol{x}|^{2}\right) \text { for } \boldsymbol{x} \rightarrow \mathbf{0}
$$

with $Q$ the solution of the Riccati equation

$$
2 \boldsymbol{Q} \boldsymbol{\sigma}(\mathbf{0}) \boldsymbol{Q}+\boldsymbol{Q} \boldsymbol{A}+\boldsymbol{A}^{T} \boldsymbol{Q}=\mathbf{0}
$$

The eikonal function $\psi(\boldsymbol{x})$ is constant on $\partial D$ with the local expansion

$$
\psi(\rho, s)=\hat{\psi}+\frac{1}{2} \rho^{2} \phi(s)+o\left(\rho^{2}\right) \text { for } \rho \rightarrow 0
$$


We find that $\boldsymbol{Q}=\boldsymbol{I}, \hat{\psi}_{\alpha}=\psi_{\alpha}(-1)=\frac{1}{2}(1-\alpha)^{2}$, and a direct integration gives

$$
\begin{aligned}
& \int_{0}^{2 \pi} K_{0}(0, s) \xi_{\alpha}(s) d s=\frac{4 \pi\left(\alpha^{4}+4 \alpha^{2}+1\right)}{C(\omega)\left(1+\alpha^{2}\right)}, \\
& C(\omega)=\frac{3 \omega}{8}-\frac{8 / \omega}{1+(4 / \omega)^{2}}+\frac{4 / \omega}{\left.4+(4 / \omega)^{2}\right)}>0 .
\end{aligned}
$$

It follows that for $(1-\alpha)^{2} / 2 \varepsilon=O(1)$, the MFPT

$$
\begin{aligned}
\bar{\tau}_{\varepsilon} & \sim \frac{C(\omega) \sqrt{2 \pi \varepsilon}(1+\alpha)^{2}}{4\left(1+4 \alpha^{2}+\alpha^{4}\right)} \exp \left\{\frac{\hat{\psi}_{\alpha}}{\varepsilon}\right\} \\
& \sim \frac{C(\omega) \sqrt{2 \pi \varepsilon}}{6} \exp \left\{\frac{(1-\alpha)^{2}}{2 \varepsilon}\right\}=O(1),
\end{aligned}
$$

so it is not exponentially long in $\varepsilon^{-1}$ as in Kramers' and classical exit problems [14, 15, 24, 25].

\subsection{The exit time distribution is well approximated using the two first eigenvalues}

The distribution of exit times $\operatorname{Pr}\left\{\tau^{\boldsymbol{x}}=t\right\}$ of the stochastic process $\boldsymbol{x}_{\varepsilon}$ (eq.14) starting at $\boldsymbol{x}$ to the limit cycle $|z|=1$ can be approximated by truncating the infinite sum 21 to the first two exponentials. Indeed,

$$
\operatorname{Pr}\left\{\tau^{\boldsymbol{x}}=t\right\}=\int_{D} p_{\varepsilon}(\boldsymbol{y}, t \mid \boldsymbol{x}) d \boldsymbol{y}
$$

and the expansion is

$$
f_{\text {etd }}(t)=\operatorname{Pr}\left\{\tau^{\boldsymbol{x}}=t\right\}=C_{0} e^{-\lambda_{0} t}+\sum_{n, m} \operatorname{Re}\left(C_{n, m} e^{-\lambda_{n, m} t}\right) .
$$

We approximate the distribution of exit time as

$$
\tilde{f}_{\text {etd }}(t)=C_{0} e^{-\lambda_{0} t}+C_{1} e^{-\omega_{1} t} \cos \left(\omega_{2} t+\phi\right),
$$

where $\lambda_{0}, \lambda_{1}$ are the first and the second eigenvalues respectively and $C_{0}, C_{1}, \phi$ are three constants. The peak oscillations are recovered and the frequencies obtained from both the numerical simulations and the analysis are in good agreement, see [15]. Indeed, with the parameter $\omega=20$, we found a numerical approximation for $k_{5}=20.5$. Approximating the first eigenvalue by the reciprocal of the MFPT $\frac{1}{\tau_{\alpha}}$ and using formula (42), we obtain $\frac{1}{\tau_{\alpha}}=1.57$ while the numerical approximation gives $\frac{1}{\tau_{\alpha}}=1.66$ and the best fit approximation gives $k_{2}=1.402$. Finally, there is also a good agreement with the first real part of the second eigenvalue: using $\omega=4$, we obtain for its approximation $\tilde{\omega}=k_{4}=4$ (see also [15] for details).

\subsection{Dynamics occurring in higher dimensions}

Although we presented here an analysis of exit time to dynamics in two dimensions, which represented a neural network described by synaptic depression, the present approach can be generalized in three dimensions and higher to study periodic bursting, which usually involves many degrees of freedom. Having critical points located close to separatrices seems to be a generic situation in theoretical neuroscience, which leads to a large diversity of behaviors. Thus the emergence of bursting activity, described by a large range of parameters [26], can be studied as an escape problem from a basin of attraction with novel metric properties between the recurrent sets. This approach has potential applications for Morris-Lecar and Fitz-Nagumo equations. 


\section{DISCUSSION AND CONCLUSION}

We have investigated here some aspect of neuronal Bursting, which is involved in information processing. It characterizes the ability of neuronal networks to transiently retain information. This process is certainly needed in brain function such as working memory and for the early process of memorization [9]. To analyze some properties of Bursting, we presented here some mathematical methods for estimating the bursting time for various parameters of the model and in particular the synaptic connection $J$.

The models we presented are quite general but assume that the neural networks are homogeneous are unstructured. For structured neural networks, such the hippocampus, cortical columns or the barrel cortex and many others, the network organization should be taken into account, requiring much more sophisticated models. However, at the level of small networks, the present analysis suggests that increasing synaptic strength, and thus the number of functional synapses between neurons, is associated with an increase of the reverberation time. Synaptic plasticity is also associated with an increase in synaptic strength, and thus in the mean number of connections. Hence the results presented here further suggest that although synaptic formation might increase linearly with $J$ in a long-term learning process, the bursting window, which characterizes buffer memory, is growing slowly and the size increases sublinearly before it reaches a maximum, where a large number of synapses are required to observe a significant change in the bursting response time.

Moreover, using a modeling approach, we have reviewed modelings and analysis of the distribution of times in the Up-state of the membrane potential, showing several oscillation peaks. These oscillations are due to the intrinsic dynamics of the synaptic depression model. The underlying peak oscillation phenomena is quite different from stochastic resonance and is a generic effect of stochastic dynamical systems where the focus point is located close enough to the boundary. The peaks frequency is independent of the noise amplitude, but depends on the amount of synaptic connections. It is conceivable that neuronal cells have found a possibility to exploit this quantification property. A possible prediction of the model is that working memory for attention could also show a quantification of the attention time, that would be interesting to investigate experimentally.

In the past 15 years, neuroscience has continuously offered novel challenges for theoreticians, pushing the development of novel methods for data analysis, constructing and analyzing multiscale models based on stochastic and partial differential equations. We have shown here that novel analytical methods can be developed. In some other cases, novel simulations tools are expected to bridge the time scales and should also be used to describe neural network behavior. In the given examples, resolving the spectrum of a non-selfadjoint operator allowed understanding the nature of oscillation for the time distribution function. Thus mathematical analysis clarifies experimental behaviors and theoretical approaches are not anymore a superficial luxury for progresses in neuroscience, but it is now an undisputable step that can lead to novel and unprecedent predictions and quantification. To conclude, neuroscience leads to novel mathematical problems where novel methods need to be developed, that will certainly refuel vigor in modern analysis. 


\section{REFERENCES}

[1] Abramowitz M and I Stegun, Handbook of Mathematical Functions with Formulas, Graphs, and Mathematical Tables, Dover Publications, NY 1972.

[2] Anderson J, Lampl I, Reichova I, Carandini M, Ferster D., Stimulus dependence of two-state fluctuations of membrane potential in cat visual cortex. Nat Neurosci. 2000;3(6):617-21.

[3] Bacci A, Verderio C, Pravettoni E, Matteoli M, Astrocytes are required for the oscillatory activity in cultured hippocampal neurons. Eur J Neurosci 1999, 11:389-397.

[4] Bart E, Bao S, Holcman D, Modeling the spontaneous activity of the auditory cortex, J Comput Neurosci. $2005 ; 19(3): 357-78$.

[5] Bender C and S Orszag Advanced Mathematical Methods for Scientists and Engineers. McGraw-Hill, New York, 1978.

[6] Bressloff P., Folias S., Front Bifurcations in an Excitatory Neural Network, SIAM Journal on Applied Mathematics,2001 Vol. 65, Number 1 pp. 131-151

[7] J. Cartailler D. Holcman, Synaptic depression-facilitation models explains the changes in neural network excitability when connexins are removed from astrocytes (pre-print) 2014.

[8] X. Chen N.Rochefort, B. Sakmann, and A. Konnerth, Reactivation of the Same Synapses during Spontaneous Up States and Sensory Stimuli, Cell 2013, 4, 31-39.

[9] Cohen D Segal M, Homeostatic presynaptic suppression of neuronal network bursts. J. Neurophysiol. $2009101,2077-2088$.

[10] Cohen D Segal M, Network bursts in hippocampal microcultures are terminated by exhaustion of vesicle pools. J. Neurophysiol.

2011, 106, 2314-2321.

[11] Compte A, Sanchez-Vives MV, McCormick DA, Wang XJ, J. Neurophysiol 2003, 89: 2707-2725.

[12] Cossart R, Aronov D, Yuste R, Attractor dynamics of network UP states in the neocortex. Nature. 2003 ;423(6937):283-8.

[13] K. Dao Duc, Lee CY, D. Cohen, M. Segal, N. Rouach, D. Holcman, Modeling bursting reverberation in small and large neuronal networks with synaptic depression-facilitation. (revision) Plos One 2014.

[14] K. Dao Duc, X. Chen, P. Parruto, J. Epsztein, A. Konnerth Holcman D., (submitted).

[15] K. Dao Duc, Z. Schuss Holcman D., SIAP, Revision 2014.

[16] Day M. V. Cycling and skewing of exit measures for planar systems. Stochastics, 1994, 48, $227-247$.

[17] Ermentrout, G. B.; Cowan, J. D. Large scale spatially organized activity in neural nets. SIAM J. Appl. Math.1980, 38, no. 1, $1-21$.

[18] Espinosa JS, Stryker MP, Development and plasticity of the primary visual cortex. Neuron. 2012;75(2):230-49.

[19] Freidlin M, Wentzell, A Random perturbations of dynamical systems. Grundlehren der Mathematischen Wissenschaften 260 (Second edition ed.). Springer-Verlag, New York, 1998.

[20] Fuster J Alexander G, Neuron activity related to short-term memory. Science 1971 173, $652-654$.

[21] Hahn T Sakmann B Mehta MR, Differential responses of hippocampal subfields to cortical up-down states. Proc Natl Acad Sci U S A. 2007;104(12):5169-74.

[22] B. Hille, Ionic Channels of Excitable Membranes. WH Freeman Ed. 2004.

[23] Holcman, D. and M. Tsodyks, The emergence of up and down states in cortical networks. PLOS Comp. Biology, $20062(3)$ :e23.

[24] Holcman D., Schuss, Z., Oscillatory survival probability and eigenvalues of non-self adjoint elliptic operators, SIAM Multiscale

Modeling and Simulations, 2014 12-3, pp. 1294-1308.

[25] Holcman D.,K. Daoduc, Schuss, Z., Oscillatory survival probability and eigenvalues of non-self adjoint elliptic operators, PRE 2014 89(3):030101.

[26] Izhikevich E J Moehlis, Dynamical Systems in Neuroscience: The geometry of excitability and bursting, SIAM review 2001, $50(2), 397$.

[27] ER Kandel, J Schwartz, T Jessell, Principles of Neural Science. McGraw-Hill Medical; 5th Revised edition, 2012.

[28] Kenet T, Bibitchkov D, Tsodyks M, Grinvald A, Arieli A., Spontaneously emerging cortical representations of visual attributes. Nature. 2003;425(6961):954-6.

[29] Lau P Bi Synaptic mechanisms of persistent reverberatory activity in neuronal networks. Proc. Natl. Acad. Sci. U.S.A., 2005, 102, 10333-10338.

[30] Maier, S., Stein, L. Limiting exit location distributions in the stochastic exit problem. SIAM J. Appl. Math.1997, 57, no. 3, $752-790$.

[31] Markram H Wang Y Tsodyks M, Differential signaling via the same axon of neocortical pyramidal neurons. Proc. Natl. Acad. Sci. 1998, 95, 5323-5328.

[32] Matkowsky B Schuss Z, On the problem of exit. Bull. Amer. Math. Soc. 1976, 82, no. 2, $321-324$.

[33] Matkowsky B, Schuss, Z, The exit problem for randomly perturbed dynamical system. SIAM J. of Appl. Math., 1977, 33, $365-382$.

[34] Matkowsky, B.J., Schuss, Z. Diffusion across characteristic boundaries. SIAM J. of Appl. Math.,1982, 42(4), 822-834.

[35] Muller, M., Goutman J., Kochubey O.\& Schneggenburger R. Interaction between facilitation and depression at a large CNS synapse reveals mechanisms of short-term plasticity. J. Neurosci. 2010, 30, 2007-2016.

[36] Miller KD, Keller JB, Stryker MP. Ocular dominance column development: analysis and simulation, Science. 1989, 11;245(4918):605-15. 
[37] Mills J, Pitman RM, Contribution of potassium conductances to a time-dependent transition in electrical properties of a cockroach motoneuron soma. J Neurophysiol 1999, 81(5): 2253-2266

[38] Oster AM, Bressloff PC, A developmental model of ocular dominance column formation on a growing cortex. Bull Math Biol. 2006;68(1):73-98.

[39] Picardo, M.A., Guigue, P., Bonifazi, P., Batista-Brito, R., Allene, C., Ribas, A., Fishell, G., Baude, A. \& Cossart, R. Pioneer GABA cells comprise a subpopulation of hub neurons in the developing hippocampus. Neuron 2011 71, 695-709.

[40] Sah P (1996) Ca(2+)-activated K+ currents in neurones: types, physiological roles and modulation. Trends Neurosci 19:150154 .

[41] Staley KJ, Longacher M, Bains JS, Yee A., Presynaptic modulation of CA3 network activity. Nat Neurosci. (1998);1(3):201-9.

[42] Traub R. , R. Miles, Neuronal Networks of the Hippocampus, Cambridge 1991.

[43] Tsodyks, M., Pawelzik, K.\& Markram H. (1998) Neural networks with dynamic synapses. Neural Comput. 10, 821-835.

[44] Tsodyks MV, Markram H. Proc Natl Acad Sci U S A. 1997;94(2):719-23. Erratum in: Proc Natl Acad Sci U S A 1997; 94(10):5495.

[45] Schuss, Z. (1980), Theory and Applications of Stochastic Differential Equations, Wiley Series in Probability and Statistics. John Wiley Sons, Inc., New York.

[46] Schuss, Z. Diffusion and Stochastic Processes: an Analytical Approach. Springer series on Applied Mathematical Sciences vol.170, 2010 Springer NY.

[47] Shu Y, Hasenstaub A, McCormick DA. Turning on and off recurrent balanced cortical activity. Nature. 2003;423(6937):288-93.

[48] Verechtchaguina T, Sokolov IM, Schimansky-Geier L. Phys Rev E Stat Nonlin Soft Matter Phys.(2006) 73:031108.

[49] Wilson HR, Cowan JD. Excitatory and inhibitory interactions in localized populations of model neurons. Biophys J. $1972 ; 12(1): 1-24$

[50] Wilson HR, Cowan JD. A mathematical theory of the functional dynamics of cortical and thalamic nervous tissue. Kybernetik $1973 ; 13(2): 55-80$.

[51] Wyart, C., Cocco, S., Bourdieu L., Léger, J.F., Herr C. \& Chatenay, D. Dynamics of excitatory synaptic components in sustained firing at low rates. J. Neurophysiol., 2005 93, 3370-80. 